\title{
POLARIMETRIA ÓPTICA E MODELAGEM DE POLARES OBSERVADAS NO OPD/LNA NO PERÍODO DE 2010-2012
}

\section{OPTICAL POLARIMETRY AND MODELING OF POLARS OBSERVED IN OPD/LNA IN THE PERIOD 2010-2012}

\author{
Karleyne M. G. Silva ${ }^{1}$ \\ Cláudia V. Rodrigues ${ }^{1}$ \\ Joaquim E. R. Costa ${ }^{1}$ \\ Deonísio Cieslinski ${ }^{1}$ \\ Leonardo A. Almeida ${ }^{1}$ \\ Victor S. Magalhães ${ }^{1}$
}

RESUMO: Neste trabalho apresentamos os primeiros resultados do estudo de uma nova amostra de 7 candidatas a polares a partir de dados polarimétricos obtidos no observatório do Pico dos Dias / LNA. Dos 4 objetos analisados até o momento, confirmamos a presença de polarização alta e variável em 3, o que indica a presença de emissão ciclotrônica e sua classificação como polares. Esses dados serão modelados utilizando-se o código CYCLOPS.

Palavras-chave: variáveis cataclísmicas; estrelas binárias; polarimetria.

ABSTRACT: In this work, we present the first results of a study of a new sample of 7 polar candidates from polarimetric data obtained at the Pico dos Dias / LNA observatory. From the four polars analysed so far, we confirm the presence of high and variable polarization in 3. The data will be analysed using the code CYCLOPS.

Keywords: cataclysmic variables; binary stars; polarimetry.

\footnotetext{
${ }^{1}$ Divisão de Astrofísica - Instituto Nacional de Pesquisas Espacias - INPE. E-mail: karleyne@gmail.com.
} 


\section{INTRODUÇÃO}

As polares (Figura 1) são estrelas binárias onde existe a transferência de plasma entre as componentes do sistema devido à grande proximidade entre elas. $\mathrm{O}$ material proveniente da estrela doadora, uma anã vermelha, é capturado pelo intenso campo magnético da estrela receptora, uma anã branca. $O$ material acumulado na superfície da anã branca forma uma região de acréscimo que emite radiação polarizada no óptico e radiação em raios $X$ (CROPPER, 1990). Essa radiação é modulada com o período orbital do sistema.

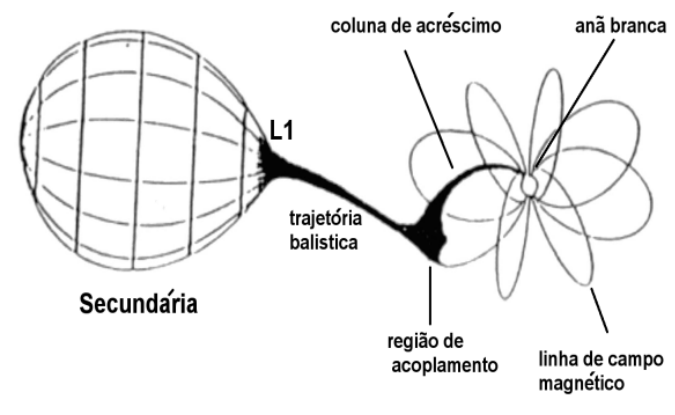

Figura 1 - Representação de uma polar e descrição das componentes do sistema. Fonte: Adaptada de Cropper (1990).

Dois catálogos online llistam as polares e candidatas conhecidas: Downes (2001) apresenta cerca de 80 polares confirmadas e Ritter e Kolb (2003) apresenta 135 sistemas, entre candidatos não confirmados e polares. Dessa forma, 55 candidatas precisam de mais informações para sua classificação. A presença de emissão polarizada confirma ocorrência de emissão ciclotrônica da coluna de acréscimo, logo sua detecção confirma a classificação de uma polar. A não detecção de polarização não descarta a classificação como polar, pois há um grupo de sistemas que apresentam valores muito baixos de polarização (AMORIM, 2011).

O estudo de uma polar permite explorar cenários particulares determinados pela configuração geométrica específica de cada fonte, como a existência de uma ou duas regiões emissoras, a ocorrência de auto-eclipses, a ocorrência de absorção por material localizado no plano orbital ou ao longo das linhas do campo magnético e a distribuição de temperaturas e densidades ao longo da região de acréscimo. O estudo de uma amostra homogênea utilizando-se uma mesma metodologia permite determinar os fatores geométricos e ao excluí-los, estudar as propriedades físicas comuns a todas as polares.

\section{DESENVOLVIMENTO}

\subsection{Descrição da amostra}

Com o objetivo de compreender os processos físicos que ocorrem em polares, nosso grupo da Divisão de Astrofísica-INPE realizou medidas polarimétricas de um conjunto de 7 polares entre 2010 e 2012 realizadas no Observarvatório do Pico dos Dias/ LNA, utilizando o módulo polarimétrico no telescópio de $1.6 \mathrm{~m}$. Estas candidatas a polares são: SWIFT J2319.4+2619, SWIFT J2218.5+1925, V393 Pav, V2301 Oph, CCS100216:1503-2207, 1RXS J1002192534 e RX J0154.0-5947. 
Tabela 1 - Descrição dos dados de nossa amostra

\begin{tabular}{|c|c|c|c|c|}
\hline Objeto & Data & Filtro & Tempo de exposição (s) & Número de imagens \\
\hline \multirow[t]{5}{*}{ SWIFT J2319.4+2619 } & $10-11 / 08 / 2010$ & $\mathrm{~V}$ & 100 & 128 \\
\hline & $11-12 / 08 / 2010$ & $\mathrm{R}$ & 60 & 144 \\
\hline & $30 / 09-01 / 10 / 2010$ & V & 90 & 83 \\
\hline & $13-14 / 10 / 2010$ & V & 90 & 125 \\
\hline & $20-21 / 09 / 2011$ & $\mathrm{R}$ & 200 & 64 \\
\hline \multirow[t]{4}{*}{ SWIFT J2218.5+1925 } & $03-04 / 11 / 2010$ & V & 60 & 144 \\
\hline & $14-15 / 06 / 2012$ & $\mathrm{R}$ & 60 & 37 \\
\hline & $15-16 / 06 / 2012$ & $\mathrm{R}$ & 60 & 96 \\
\hline & $16-17 / 06 / 2012$ & I & 60 & 136 \\
\hline \multirow[t]{6}{*}{ V393 Pav } & $09-10 / 08 / 2010$ & I & 80 & 144 \\
\hline & $10-11 / 08 / 2010$ & V & 80 & 176 \\
\hline & $11-12 / 08 / 2010$ & $\mathrm{R}$ & 100 & 128 \\
\hline & $08-09 / 10 / 2010$ & $\mathrm{R}$ & 90 e 120 & 73 \\
\hline & $13-14 / 10 / 2010$ & V & 80 & 96 \\
\hline & $14-15 / 10 / 2010$ & I & 60 e 90 & 111 \\
\hline \multirow[t]{5}{*}{ CCS100216:1503-2207 } & $15-16 / 06 / 2012$ & $\mathrm{R}$ & 60 & 80 \\
\hline & $16-17 / 06 / 2012$ & $\mathrm{R}$ & 60 & 100 \\
\hline & $16-17 / 06 / 2012$ & $\mathrm{~V}$ & 60 & 128 \\
\hline & $17-18 / 06 / 2012$ & i & 50 & 160 \\
\hline & $17-18 / 06 / 2012$ & V & 50 & 129 \\
\hline \multirow{2}{*}{$1 \mathrm{RXS} J 1002-192534$} & $24-25 / 04 / 2012$ & $\mathrm{~V}$ & 60 & 176 \\
\hline & $25-26 / 04 / 2012$ & I & 90 & 161 \\
\hline \multirow[t]{6}{*}{ RX J0154.0-5947 } & $06-08 / 08 / 2010$ & V & 30 & 97 \\
\hline & $07-08 / 08 / 2010$ & V & 30 & 144 \\
\hline & $08-09 / 08 / 2010$ & I & 40 & 160 \\
\hline & $10-11 / 08 / 2010$ & V & 40 & 53 \\
\hline & $02-03 / 11 / 2010$ & $\mathrm{R}$ & 30 & 240 \\
\hline & $03-04 / 11 / 2010$ & 1 & 30 & 128 \\
\hline \multirow[t]{4}{*}{ V2301 Oph } & $06-07 / 08 / 2010$ & $\mathrm{I}$ & 60 & 96 \\
\hline & $07-08 / 08 / 2010$ & I & 60 & 121 \\
\hline & $08-09 / 08 / 2010$ & $\mathrm{R}$ & 30 e 60 & 160 \\
\hline & $09-10 / 08 / 2010$ & I & 50 & 160 \\
\hline
\end{tabular}

A Tabela 1 apresenta o resumo das observações obtidas. Esses novos dados polarimétricos permitirão confirmar a classificação como polar. Nos casos confirmados, será realizada a modelagem da região de acréscimo com o código CYCLOPS. O CYCLOPS é um software que permite a modelagem de dados polarimétricos do óptico e de raios $X$ conjuntamente, fornecendo a determinação de parâmetros físicos e geométricos de polares (COSTA; RODRIGUES, 2009; SILVA et al., 2013).

\subsection{Polarimetria}

O material ionizado capturado pelo campo magnético da anã branca realiza uma trajetória espiral ao longo das linhas de campo magnético. A emissão é polarizada devido ao processo de emissão ciclotrônica. Quando se observar radiação de material em movimento ao longo das linhas de campo magnético, tem-se polarização circular. Quando se observa o movimento perpendicularmente ao campo magnético tem-se polarização linear. À medida que a linha de visada do sistema muda, devido ao movimento próprio da binária, a polarização varia de uma para outra. Como algumas polares apresentam cerca de $50 \%$ de emissão polarizada, elas estão entre os objetos mais polarizados do céu.

O polarimetro utilizado nas observações, descrito em Magalhães et al. (1996), possui um prisma de calcita, que divide o feixe incidente em dois feixes com polarização perpendicular entre si e uma lâmina retardadora que altera a fase entre os dois feixes, incluindo uma modulação no sinal recebido quando se modifica o ângulo de posição da lâmina. Conforme apresentado em Rodrigues, Cieslinski e Steiner (1998), a intensidade das duas imagens geradas, ordinária $\left(\mathrm{I}_{\mathrm{o}}\right)$ e extraordinária $\left(\mathrm{l}_{\mathrm{e}}\right)$, relaciona-se com os parâmetros de Stokes I, Q, U e V do feixe 
incidente através da relação estabelecida por Serkowski (1974):

$2 \mathrm{I}_{\mathrm{e}, \mathrm{o}}=\mathrm{I}+\mathrm{Q} \cos ^{2} 2 \Psi_{\mathrm{i}} \pm$

$U \operatorname{sen} 2 \Psi_{i} \cos 2 \Psi_{i} \pm V \operatorname{sen} 2 \Psi_{i}$,

onde i varia de 1 a 16 e representa a posição que o retardador assume durante as observações. Os valores $\psi_{i}$ e $\left.\right|_{e, 0}$ são conhecidos, estes últimos dois medidos a partir de fotometria de abertura das imagens. Os parâmetros $Q, U$ e $V$ são, então, expressos por:

$Q=\frac{1}{3} \sum_{i=1}^{8} \quad X_{i} \cos ^{2} 2 \Psi_{i}$,

$\mathrm{U}=\sum_{\mathrm{i}=1}^{8} \mathrm{X}_{\mathrm{i}} \operatorname{sen}^{2} 2 \Psi_{\mathrm{i}} \cos 2 \Psi_{\mathrm{i}}$ e

$V=\frac{1}{4} \sum_{i=1}^{8}=1 \quad X_{i} \operatorname{sen}^{2} 2 \Psi_{i}$.

O grau de polarização linear $(p)$ e 0 grau de polarização circular (v) são dados por:

$\mathrm{p}=\frac{\mathrm{Q}^{2}+\mathrm{U}^{2}}{\mathrm{l}^{2}}$ e $\quad \mathrm{v}=\frac{\mathrm{v}}{\mathrm{l}}$.

O ângulo de polarização linear $(\theta)$ e a incerteza associada $\left(\sigma_{\theta}\right)$ são estimados a partir de:

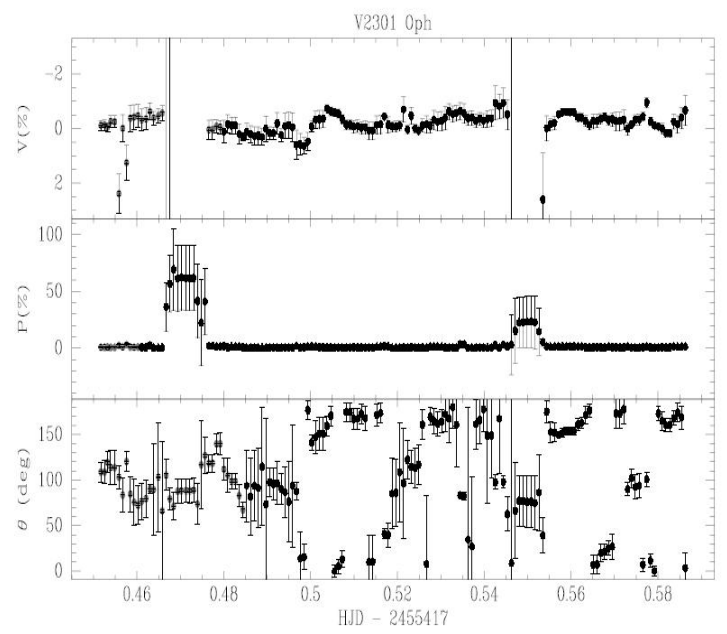

$\theta=\frac{1}{2} \operatorname{tg}-1 \frac{U}{Q}$ e $\sigma_{\theta}=28,65 \frac{\sigma_{p}}{p}$.

\subsection{Resultados da redução}

As Figuras 2 e 3 apresentam as curvas de polarização das 4 polares analisadas até agora. V2301 Oph é uma polar eclipsante. Pode-se ver que sua polarização circular é consistente com zero, considerando as barras de erros (Figura 2, à esquerda). A polarização linear também é baixa, próxima de zero. Os pontos com erros grandes referem-se a medidas feitas durante o eclipse, quando o fluxo do objeto diminui bastante. De nossa amostra, V2301 Oph era o único sistema confirmado como polar via espectroscopia e o estudo em raios $X$, no entanto, não apresenta polarização. Polares com esse comportamento foram estudadas por Amorim (2011).

V393 Pav apresentou polarização circular alta, variando de -22 a 3\% (Figura 2, à direita). Foram cobertos cerca de três períodos orbitais na banda $R$. A polarização linear é relativamente baixa. A redução de dados de outros filtros permitirá refinar a efemérides desta nova polar.

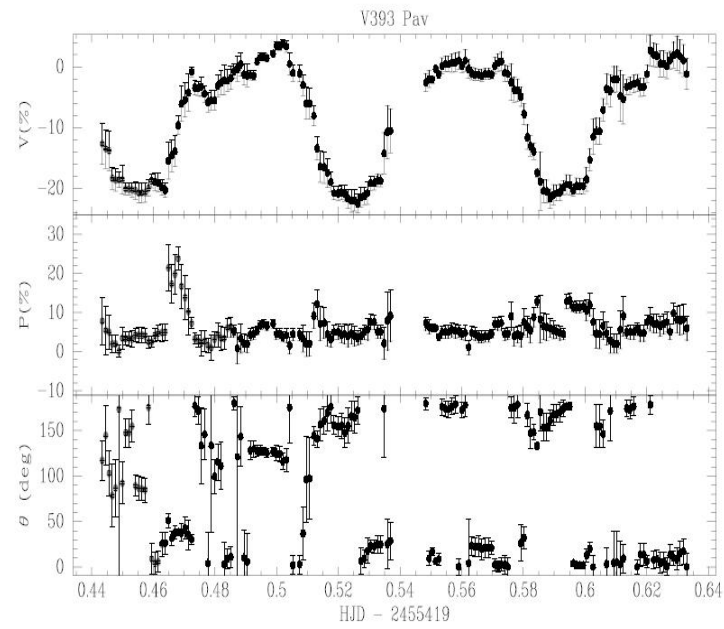

Figura 2 - Curva de polarização de V2301 Oph e V393 Pav. De cima para baixo, polarização circular (v), polarização linear (p) e ângulo da polarização linear $(\theta)$. 

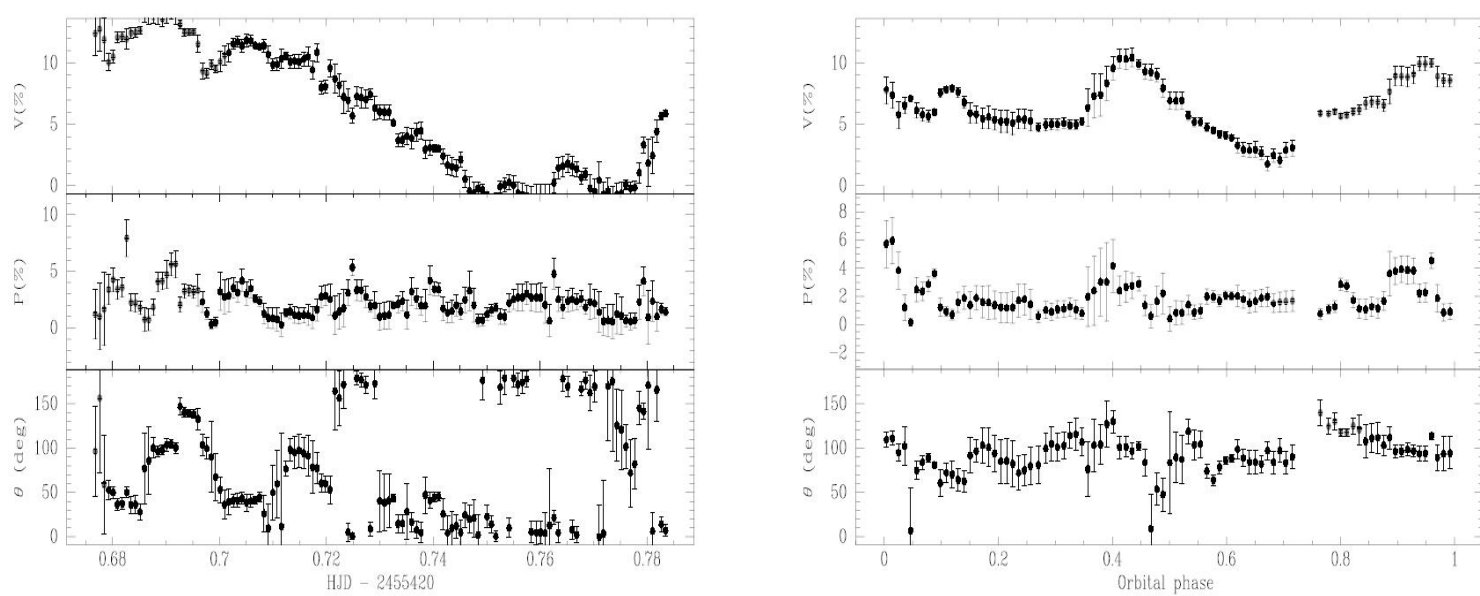

Figura 3 - Curva de polarização de SWIFT J2319.4+2619 à esquerda e RX J0154.0-5947, à direita. De cima para baixo, polarização circular (v), polarização linear (p) e ângulo da polarização linear $(\theta)$.

SWIFT J2319.4+2619, conforme mostrado na Figura 3 à esquerda, apresenta polarização circular variando de 0 a $15 \%$, os dados que analisamos não cobrem o período orbital completo. A polarização linear é baixa, chegando, no máximo, a $5 \%$.

RX J0154.0-5947, conforme apresentado na Figura 3 à direita, apresenta polarização circular variando de 2 a 13\%, nunca chegando a zero. Foi feito um diagrama de fase utilizando-se a efeméride de Ramsay e Cropper (2004). Foi observado, aproximadamente, um período orbital completo. A polarização linear também é baixa, com valor médio de $4 \%$.

\section{CONCLUSÕES}

Apresentamos aqui os primeiros resultados deste novo projeto, que consistiu na polarimetria de uma nova amostra. Verificamos a presença de polarização alta e variável em três das quatro polares analisadas até 0 momento. Esses resultados inserem-se em um projeto maior para determinação dos parâmetros geométricos e físicos de um grupo de polares de forma homogênea usando o CYCLOPS.

\section{REFERÊNCIAS}

AMORIM, R. R. Candidatos a variáveis cataclísmicas magnéticas com polarização baixa. 2011. 123 p. Dissertação (Mestrado) Instituto Nacional de Pesquisas Espaciais, São José dos Campos, 2011.

COSTA, J. E. R.; RODRIGUES, C. V. Stokes imaging of AM Her systems using 3D inhomogeneous models - I. Description of the code and an application to V834 Cen. Monthly Notices of the Royal Astronomical Society, v. 398, p. 240-248, Sep. 2009.

CROPPER, M. The polars. Space Science Reviews, v. 54, p. 195-295, Dec. 1990.

DOWNES, R. A Catalog and Atlas of Cataclysmic Variables: The Living Edition. Astronomical Society of the Pacific, v. 113, n. 784, p. 764-768, jun. 2001.

MAGALHÃES, A. M. et al. High Precision CCD Imaging Polarimetry. Polarimetry of the interstellar medium. Astronomical Society of 
the Pacific Conference Series, v. 97, p. 118- Astronomy and Astrophysics, v. 335, p. 979122, 1996.

984, jul. 1998.

RAMSAY, G.; CROPPER, M. The energy balance of polars revisited. Monthly Notices of the Royal Astronomical Society, v. 347, n. 2, p. 497-507, Jan. 2004.

RITTER, H.; KOLB, U. Catalogue of cataclysmic binaries, low-mass X-ray binaries and related objects. Astronomy and Astrophysics, v. 404, p. 301-303, Jun. 2003.

RODRIGUES, C. V.; CIESLINSKI, D.; STEINER, J. E. Polarimetry and spectroscopy of the polar RX J1141.3-6410.
SERKOWSKI K. Polarimeters for Optical Astronomy. In: GEHRELS, T. (eds.) Planets, Stars, and Nebulae Studied with Photopolarimetry. Arizona, Univ. Arizona Press, 1974. p. 135.

SILVA, K. M. G. et al. Stokes imaging of AM Her systems using 3D inhomogeneous models - II. Modelling X-ray and optical data of CP Tucanae. Monthly Notices of the Royal Astronomical Society, v. 432, p. 1587-1599, 2013. 